\title{
Dynamic stability of spindles controlled by molecular motor kinetics
}

\author{
Otger Campàs, ${ }^{1,2}$ Jaume Casademunt, ${ }^{2}$ and Ignacio Pagonabarraga ${ }^{3}$ \\ ${ }^{1}$ Institut Curie, UMR 168, 26 rue d'Ulm, F-75248 Paris Cedex 05, France \\ ${ }^{2}$ Departament d'ECM, Universitat de Barcelona, Avinguda Diagonal 647, E-08028 Barcelona, Spain \\ ${ }^{3}$ Departament de Física Fonamental, Universitat de Barcelona, \\ Avinguda Diagonal 647, E-08028 Barcelona, Spain
}

\begin{abstract}
We analyze the role of the force-dependent kinetics of motor proteins in the stability of antiparallel arrays of polar filaments, such as those in the mitotic spindle. We determine the possible stable structures and show that there exists an instability associated to the collective behavior of motors that leads to the collapse of the spindle. Our analysis provides a general framework to understand several experimental observations in eukaryotic cell division.
\end{abstract}

PACS numbers: 87.16.Ac, 87.16.Nn, 87.16.Ka, 05.45.-a

Living cells display several structures that arise from the self-organization of polar filaments and motor proteins 1]. Several in vitro studies have shown that mixtures of kinesin motors and microtubules (MTs) can spontaneously develop complex spatio-temporal patterns [2]. These self-organization processes are essential for eukaryotic cell division [3]. During mitosis, motor proteins organize MTs in a bipolar structure, the mitotic spindle, which serves as a scaffold to transmit the necessary forces for chromosome segregation [4]. The spindle consists of two MT asters that overlap in the central region. The MTs, with their minus-ends located at the aster poles, are crosslinked by many different motor proteins 3 3, 5]. One particular type of motors, the plus-ended bipolar kinesins (e.g. Eg5 or Klp61F), has been shown to be essential for the spindle stability. A decrease in their concentration below a certain threshold causes the spindle collapse [6, 7], and their total inhibition prevents bipolar spindle formation [8]. In addition, Eg5 motors have been shown to drive the MT poleward flux [7] and homolog motors to induce the formation of (interpolar) MT bundles [9].

Bipolar motors are composed of two connected units, each one composed of two motor domains. Both units can move simultaneously and independently on MTs [10]. These motors are able to crosslink MTs [9] and slide them with respect to each other when they are in an antiparallel configuration [10], like in the central region of the spindle (Fig. [1 $\mathrm{l}, \mathrm{c})$. As a result, these motors produce an outward force along the spindle axis and generate a MT flux toward the poles [7]. Typical forces involved in mitosis lay in the nanoNewton range [11]. Since individual motors cannot exert forces larger than a few picoNewtons, their collective action is required to ensure the stability of the mitotic spindle. At metaphase, this dynamic structure reaches a steady state with MTs of nearly constant length undergoing permanent treadmilling [7, 12], polymerizing at the + end and depolymerizing at the end.

The theoretical study of motors and MT mixtures has been recently addressed using continuum coarse grained

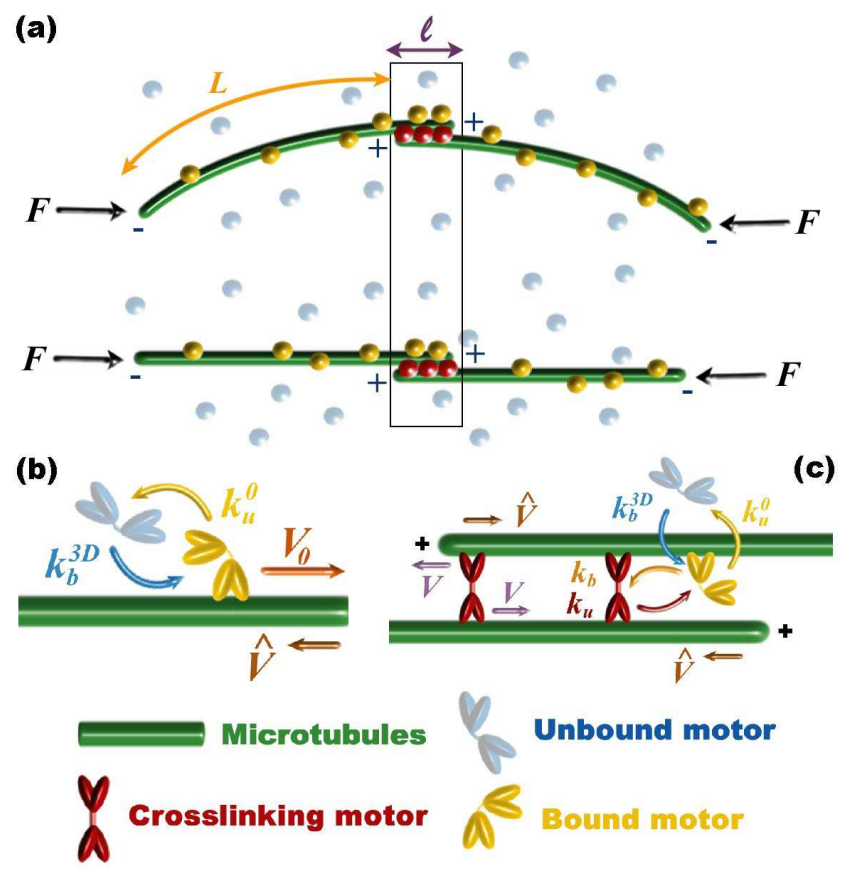

FIG. 1: (a) Antiparallel array of MTs under the action of a longitudinal force $F$. Buckled and non-buckled arrays are shown. The minus and plus ends of MTs are depicted as and + respectively. Motors are represented by dots. In the non-overlapping region of length $L$ there are no antiparallel filaments and the motors are not subject to any force. The motors in the overlapping region of length $\ell$ sustain the structure by crosslinking and sliding antiparallel filaments. (b-c) Possible kinetic events of a motor in the non-overlapping region (b) and in the overlapping region (c). The velocities $V$ and $\hat{V}$ are the crosslinking motor velocity and the MT poleward velocity respectively.

descriptions [13, 14], which have elucidated their basic self-organizing principles. However, the coupling between force-dependent motor kinetics and local forces in selforganized structures has not been addressed. In this Letter, we study the dynamic stability of antiparallel arrays of MTs under the action of longitudinal forces, in the 
presence of molecular motors able to collectively hold the structure by stochastically crosslinking the filaments. We analyze the effects of the motor kinetics on the stability of the structure, and show that several phenomena observed in eukaryotic cell division can be understood on the same physical basis.

In order to comprehend the basic physical mechanisms controlling the stability of a spindle, we concentrate on a simplified geometry. We consider a pair of antiparallel MTs (or an antiparallel MT bundle) of fixed length, under the action of an inward force $F$ (Fig. 11). In the steady state, there is a region of length $\ell$ where the antiparallel filament array overlaps (overlapping region). The motors in this region can crosslink antiparallel MTs and slide them in opposite directions, generating an outward force that balances the applied force $F$. We assume the antiparallel MT sliding to be the only mechanism generating the poleward MT flux, as suggested experimentally [7]. Out of this overlapping region there are two regions of length $L$ (non-overlapping regions; see Fig. 11a) where motors cannot apply forces to sustain the spindle. Since we concentrate on the spindle stability and do not address the mechanisms that determine $L$ and $\ell$, we take them as given parameters.

The motors in the non-overlapping region can be either bound to a MT or freely diffusing in the bulk (Fig. 1b). We assume a constant bulk motor density, $\rho_{3 D}$ [15], and consider the motors in the bulk to attach onto MTs at a rate $k_{b}^{3 D}$. Once bound to a MT, a motor moves convectively with a mean velocity $V_{0}$ toward the plus-end of the MT and detaches at a rate $k_{u}^{0}$. At mean field level, the dynamics of the bound motor density, $\rho(s, t)$, can then be expressed as [16, 17]

$$
\partial_{t} \rho(s, t)+\partial_{s} J(s, t)=-k_{u}^{0} \rho(s, t)+k_{b}^{3 D} \rho_{3 D},
$$

where $s$ is the position along the MT as measured from the MT minus ends and $J(s, t)$ is the flux of bound motors. For simplicity, we assume the bound motors to be in a low density phase and write $J(s, t)=\rho(s, t)\left(V_{0}-\hat{V}\right)$ in the laboratory reference frame, with $\hat{V}$ being the velocity of MTs toward the poles (Fig. 10,c).

In the central overlapping region, bipolar motors can be either in a crosslinking state or in a bound state. In the former state both motor units are attached to a pair of antiparallel MTs, sliding them in opposite directions and supporting a fraction of the total force $F$. As a result, the crosslinking motors move with a forcedependent velocity $V$ (Fig. 11). Based on experimental observations [18], we write a linear force-velocity relation, $V=V_{0}\left(1-f_{m} / f_{s}\right)$, where $f_{m}$ is the load applied on the motor and $f_{s}$ its stall force. We consider a number $n_{c}$ of independent crosslinking motors to equally share the total applied force, so that $f_{m}=F / n_{c}$. As the poleward MT movement is driven only by these motors, we identify $\hat{V}=V$ (Fig. 11). Each unit of a motor in the crosslinking state can detach at a force-dependent rate $k_{u}\left(f_{m}\right)=k_{u}^{0} \exp \left(f_{m} b / K_{B} T\right)$ (Kramers theory [19]), where $b$ is a length in the nanometer scale characterizing the activated process and $K_{B} T$ the thermal energy. Such exponential sensitivity to applied load has indeed been observed experimentally 20]. After the detachment of one motor unit, the bipolar motor is only bound to one MT and unable to apply force. Such motor can either detach the bound motor unit left at a rate $k_{u}^{0}$ and diffuse into the bulk, or re-attach the unbound motor unit at a rate $k_{b}$ and become a crosslinking motor again. The motors in the bulk can also attach directly to the MTs in the overlapping region at a rate $k_{b}^{3 D}$.

The relevant variables being the number of motors sustaining the spindle, we neglect their spatial distribution in the overlapping region. Accordingly, the equations for the average number of crosslinking and bound motors, $n_{c}$ and $n_{b}$ respectively, read

$$
\begin{aligned}
& \frac{d n_{c}}{d t}=k_{b} n_{b}-k_{u}\left(n_{c}\right) n_{c}, \\
& \frac{d n_{b}}{d t}=2 J(L, t)+k_{b}^{3 D} \rho_{3 D} \ell+k_{u}\left(n_{c}\right) n_{c}-\left(k_{u}^{0}+k_{b}\right) n_{b},
\end{aligned}
$$

where $J(L, t)$ is the convective flux of bound motors coming from a non-overlapping region, with $L$ being the arclength of a MT from its pole to the overlapping region (Fig. 13). The value of $J(L, t)$ is determined by the solution of Eq. 1] When the motor processivity length, $l_{p} \equiv V_{0} / k_{u}^{0}$, is smaller than the characteristic spindle length $\left(l_{p} \ll L[21]\right)$, the flux $J(L, t)$ is determined by a constant bound motor density, $\rho=k_{b}^{3 D} \rho_{3 D} / k_{u}^{0}$, fixed by the exchange of motors with the bulk. In this case the dynamics of $n_{c}$ and $n_{b}$ are decoupled from $\rho$ close to the pole.

The existence of antiparallel MT arrays under an external load $F$ is determined by the balance between motor attachment and detachment fluxes, as given by the steady state solutions, $\left\{n_{c}^{f}, n_{b}^{f}\right\}$, of Eqs. 11 2] which read

$$
\begin{aligned}
& \delta \equiv \frac{\rho_{3 D} k_{b}^{3 D} l_{p}}{\tilde{F} \alpha k_{u}^{0}} \gamma=\frac{\exp \left(1 / \tilde{n}_{c}^{f}\right)\left(\tilde{n}_{c}^{f}\right)^{2}}{2\left[1-\exp \left(-\tilde{L} \tilde{n}_{c}^{f}\right)\right]+\tilde{\ell}_{n}^{f}}, \\
& \tilde{n}_{b}^{f}=\frac{\exp \left(1 / \tilde{n}_{c}^{f}\right) \tilde{n}_{c}^{f}}{\gamma} .
\end{aligned}
$$

The quantities $\tilde{n}_{c} \equiv n_{c} / \tilde{F}$ and $\tilde{n}_{b} \equiv n_{b} / \tilde{F}$ are the normalized numbers of crosslinking and bound motors respectively. The ratio, $\tilde{F} \equiv F b / K_{B} T$, between the force $F$ and the characteristic detachment force $K_{B} T / b$ sets the natural scale of motors. The relevant dimensionless lengths are $\tilde{L} \equiv \alpha L / l_{p}$ and $\tilde{\ell} \equiv \alpha \ell / l_{p}$, and the parameter $\alpha \equiv f_{s} b / K_{B} T$ quantifies the sensitivity of motor detachment to force. The asymmetry in motor attachment/detachment events at vanishing load is characterized by $\gamma \equiv k_{b} / k_{u}^{0}$.

There always exists a critical value, $\delta_{m}$, below which there are no solutions of Eq. 3. This situation corresponds to an attachment flux of crosslinking motors that 

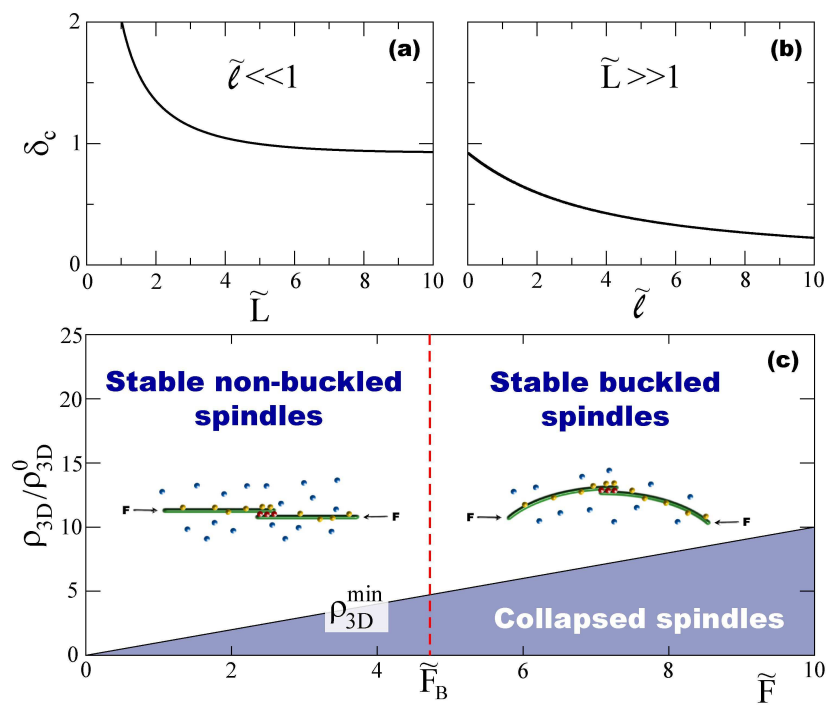

FIG. 2: (a-b) Dependence of the critical value $\delta_{c}$ on $\tilde{L}$ and $\tilde{\ell}$ in the limiting cases where (a) $\tilde{\ell} \ll 1$ and (b) $\tilde{L} \gg 1$ respectively. (c) Possible spindle structures as the bulk motor density $\rho_{3 D}$ and the force $F$ are varied $\left(\rho_{3 D}^{0} \equiv \delta_{c} \alpha\left(k_{u}^{0}\right)^{3} / k_{b}^{3 D} V_{0} k_{b}\right.$ sets the density units). Above $\rho_{3 D}^{\min }(F)$, buckled (straight) stable spindles exists for $F>F_{B}\left(F<F_{B}\right)$. Below $\rho_{3 D}^{\min }(F)$ no stable spindles exist.

can not balance their detachment flux, leading to the loss of all crosslinking motors and inducing the spindle collapse. Associated to the critical point $\delta_{m}$, there is a minimum number of crosslinking motors, $\tilde{n}_{c}^{m}$, necessary to sustain a spindle, whose value is given implicitly by

$$
\begin{aligned}
& 1+\left[\tilde{n}_{c}^{m}\left(2+\tilde{L} \tilde{n}_{c}^{m}\right)-1\right] \exp \left(-\tilde{L} \tilde{n}_{c}^{m}\right)= \\
& \tilde{n}_{c}^{m}\left[2+\frac{\tilde{\ell}}{2}\left(\tilde{n}_{c}^{m}-1\right)\right] .
\end{aligned}
$$

The actual value of $\delta_{m}$ is obtained by substituting $\tilde{n}_{c}^{f}$ in Eq. 3 by the solution of Eq. [.

In order to determine the stability of the structures, we perform a linear stability analysis of the solutions of Eq. 3. For $L \gg l_{p}$ it can be shown that the fluctuations in $\rho(s)$ are negligible and the spindle stability depends only on the dynamics of $n_{c}$ and $n_{b}$ 22]. Stable spindles exist above a critical value $\delta_{c}$. The precise expression for this critical point $\delta_{c}$ depends on the ratio between motor attachment/detachment rates at vanishing load. For $\gamma>\gamma_{c} \equiv \exp \left(1 / \tilde{n}_{c}^{m}\right)\left(1-\tilde{n}_{c}^{m}\right) / \tilde{n}_{c}^{m}-1$, the transition from an unstable array to a stable spindle corresponds to a saddle-node bifurcation at $\delta_{c}=\delta_{m}$. On the other hand, if $\gamma<\gamma_{c}$, this transition corresponds to a global bifurcation (saddle-connection 23]) at a value $\delta_{c}>\delta_{m}$. Regardless the value of $\gamma$, the same qualitative scenario is observed as $\delta$ is varied and we restrict the following discussion to the regime $\gamma>\gamma_{c}$ without loss of generality. The threshold value, $\delta_{c}$, is fixed only by $\tilde{L}$ and $\tilde{\ell}$. Typi-
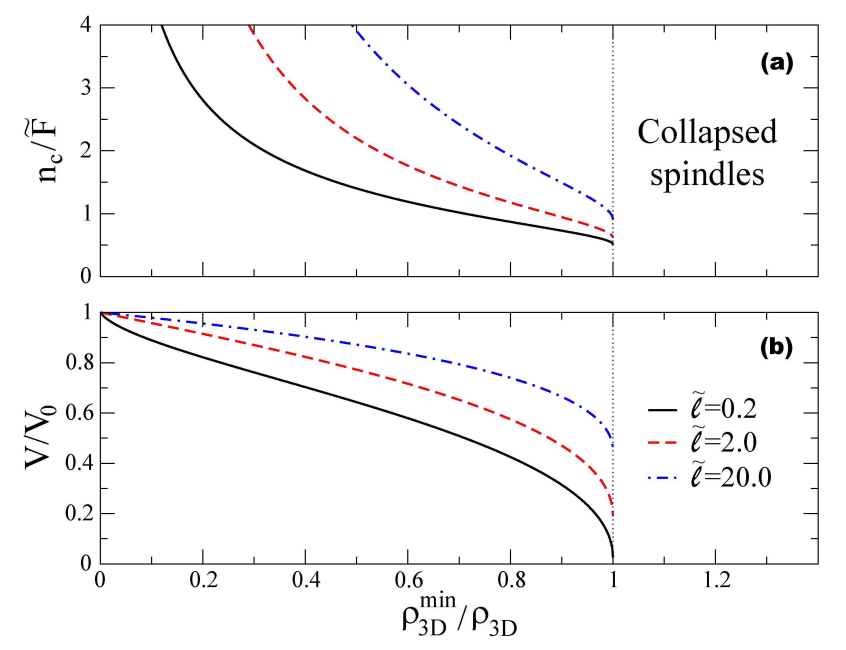

FIG. 3: Steady state analytical solutions for (a) the number of crosslinking motors and (b) the velocity of the crosslinking motors (sliding velocity), as a function of the bulk density of motors. The different curves represent different values of $\tilde{\ell}$ in the limiting regime $\tilde{L} \gg 1$.

cally $L \gg l_{p}$ and $\delta_{c}$ becomes independent of $L$ (Fig. $2 \mathrm{l}$ ), while $\ell$ modifies $\delta_{c}$ slightly (Fig. 2b). In this limit, the spindle morphology and its stability are decoupled. As the motor properties and the lengths $L$ and $\ell$ are difficult to modify experimentally, $\delta$ appears as the natural control parameter for the spindle stability, since it depends both on the applied force, $F$, and the bulk motor density, $\rho_{3 D}$.

The existence of a critical value $\delta_{c}$ implies that for a MT array under the action of a load, $F$, there exists a minimal motor bulk concentration, $\rho_{3 D}^{\min }$, below which no stable spindles are found. Using the definition of $\delta$ (Eq. 3), this minimal motor density reads

$$
\rho_{3 D}^{\min }=\frac{k_{u}^{0}}{k_{b}^{3 D} l_{p}} \frac{\alpha \delta_{c}}{\gamma} \tilde{F} .
$$

When $\rho_{3 D}>\rho_{3 D}^{\min }$ the stable spindle may be either straight or buckled depending on the value of the compressive force, $F$. For forces below (above) the buckling force $F_{B}=(\pi / 2)^{2} B / L^{2}$ of the structure ( $B$ being its bending rigidity), the stable spindle is straight (buckled). In Fig. 22 we plot the structures that can be found as the bulk density of motors and the force applied on the spindle are varied. Indeed, recent experimental observations have shown that the progressive inhibition of Eg5 motors leads to the collapse of the spindle at a finite bulk motor density [7]. Moreover, the total inhibition of homolog motors (Klp61F) has been shown to prevent bipolar spindle formation in vivo [8], in accordance with our predictions.

Above the threshold density $\rho_{3 D}^{\min }$, there is a finite amount of crosslinking motors $n_{c}$ collectively holding the spindle. In Fig. 3a we plot the stable solutions of Eq. 3] as 
a function of the bulk concentration of motors. Increasing values of $\rho_{3 D}$ and $\ell$ leads to larger motor attachment fluxes, that result in a larger amount of crosslinking motors. For a living cell in native conditions, the MTs in the mitotic spindle are typically buckled [4]. Therefore, the force applied on the MTs is of order $F_{B}$ which, for single MTs ( $5 \mu m$ in length) is about $1 p N$. Using this value for the force $F$, the number of crosslinking motors leading to a stable antiparallel array turns out to be very small $(\simeq 2)$. In this case fluctuations would dominate and, although stable arrays could be transiently formed, their lifetime would be too short (on the time scale of motor detachment). Since the buckling force of a MT bundle can be at least one order of magnitude larger [24], stable interpolar MT bundles require tenths of crosslinking motors and provide robust spindles with lifetimes over the time scale of the division process. Interpolar MT bundles are indeed observed in several organisms during cell division $9,25,26]$.

The speed of the MT flux toward the poles is determined by the MT sliding velocity, $\hat{V}$, given by the velocity of the crosslinking motors. In Fig. 3] we represent this sliding velocity as a function of $\rho_{3 D}$, for different values of $\tilde{\ell}$. It decreases from its maximal value $V_{0}$ as the bulk motor density is decreased, and it is typically finite for the minimal density $\rho_{3 D}^{\min }$ at which the spindle collapses, as observed experimentally []. At high motor concentrations $\rho_{3 D} \gg \rho_{3 D}^{\min }$, the crosslinking motors move nearly at their maximal velocity $V_{0}$ and the MTs move poleward at this velocity consequently. As the motors in the nonoverlapping regions move at velocity $V_{0}$ with respect to the MTs in the spindle (Fig.10), they appear static in the laboratory reference frame. This apparent motor stillness has indeed been observed experimentally 27]. Our analysis predicts that decreasing the bulk motor density $\rho_{3 D}$ would allow the observation of motor movement in the spindle. This observation would provide further insight on the understanding of the mitotic spindle structure.

The present approach highlights the importance of force-dependent motor kinetics on the self-organization of MTs and motors. In particular, we show that motor kinetics is a key factor in the stability of spindle-like structures under applied load. Moreover, we have developed a framework through which several observations in eukaryotic cell division, namely the spindle collapse, the MT poleward flux, the static appearance of motors in the spindle, and the existence of MT bundles, can be explained on a common physical ground. The observation of motor motion upon a decrease of motor density, as predicted here, would provide strong evidence for the self-organization of motors and MTs as the underlying principle of mitotic spindle assembly.

We thank K. Kruse, J.-F. Joanny and J. Prost for stimulating discussions. We thank the European Commission (HPRN-CT-2002-00312) and the Spanish M.E.C. (BQU2003-05042-C02-02 and FIS2005-01299) for finan- cial support. I.P. also thanks the Distinció de la Generalitat de Catalunya (Spain) for financial support.

[1] B. Alberts et al., The Molecular Biology of the Cell (Garland, New York, 2002).

[2] F.J. Nedelec, T. Surrey, A.C. Maggs, S. Leibler, Nature 389, 305 (1997).

[3] T. Wittmann, A. Hyman, A. Desai, Nat. Cell Biol. 3, E28 (2001).

[4] T.J. Mitchison, E.D. Salmon, Nat. Cell Biol. 3, E17 (2001).

[5] D.J. Sharp, G.C. Rogers, J.M. Scholey, Nature 407, 41 (2000).

[6] T.M. Kapoor, T.U. Mayer, M.L. Coughlin, T.J. Mitchison, J. Cell Biol. 150, 975 (2000).

[7] D.T. Miyamoto et al., J. Cell Biol. 167, 813 (2004).

[8] G. Goshima, R.D. Vale, J. Cell Biol. 162, 1003 (2003).

[9] D.J. Sharp et al., J. Cell Biol. 144, 125 (1999).

[10] L.C. Kapitein et al., Nature 435, 114 (2005).

[11] R.B. Nicklas, J. Cell Biol. 97, 542 (1983).

[12] T.J. Mitchison et al., Mol. Biol. Cell 15, 5603 (2004).

[13] K. Kruse, F. Jülicher, Phys. Rev. Lett. 85, 1778 (2000).

[14] K. Kruse, J.-F. Joanny, F. Jülicher, J. Prost, K. Sekimoto, Phys. Rev. Lett. 92, 078101 (2004).

[15] This is reasonable for typical spindle lengths $\left(L_{s} \sim 5 \mu \mathrm{m}\right)$ as the motor bulk concentration equilibrates over time scales, of order $\sim L_{s}^{2} / D \simeq 1 s\left(D \sim 10 \mu m^{2} s^{-1}\right.$ being the diffusion constant of the motors in the bulk), shorter than the time scale of convective motor movement along MTs, of order $\sim L_{s} / V_{0} \simeq 100 s$, for typical values of the motor velocity $\left(V_{0} \simeq 33 \mathrm{nms}^{-1}\right.$ for Eg5 10] $)$.

[16] R. Lipowsky, S. Klumpp, T.M. Nieuwenhuizen, Phys. Rev. Lett. 87, 108101 (2001).

[17] A. Parmeggiani, T. Franosch, E. Frey, Phys. Rev. Lett. 90, 086601 (2003).

[18] M.J. Schnitzer, K. Visscher, S.M. Block, Nat. Cell Biol. 2, 718 (2000).

[19] N.G. Van Kampen, Stochastic Processes in Physics and Chemistry (North Holland, Amsterdam, 2004).

[20] For non-processive myosin motors: C. Veigel, E.S. Molloy, S. Schmitz, J. Kendrick-Jones, Nat. Cell Biol. 5, 980 (2003); for processive kinesin motors: Ref. [18].

[21] Typical values for $L$ are about several microns long for most cell types, while Eg5 motors are not very processive, $l_{p}<100 \mathrm{~nm}$ [10].

[22] For $L \ll l_{p}$ the stability scenarios remain qualitatively unchanged.

[23] J. Guckenheimer, P. Holmes, Nonlinear Oscillations, Dynamical Systems, and Bifurcations of Vector Fields (Applied Mathematical Sciences, Vol. 42, Springer-Verlag, 1990).

[24] Assuming the bending rigidity of a MT bundle to scale lineraly with the number of MTs in the bundle, the buckling force of an interpolar MT bundle may be of tenths of picoNewtons.

[25] D.N. Mastronarde, K.L. McDonald, R. Ding, J.R. McIntosh, J. Cell Biol. 123, 1475 (1993).

[26] M. Winey et al., J. Cell Biol. 129, 1601 (1995).

[27] T.M. Kapoor, T.J. Mitchison, J. Cell Biol. 154, 1125 (2001). 